\title{
A rare case of asymptomatic rupture of the uterine horn in the full-term pregnancy as a consequence of laparoscopic salpingectomy
}

\author{
Seweryn Trojanowski, Anna Słomka, Paweł Stanirowski, Krzysztof Cendrowski, \\ Włodzimierz Sawicki
}

Department of Obstetrics, Gynecology and Gynecologic Oncology, Second Faculty of Medicine, Medical University of Warsaw, Poland

A 29-year-old nulliparous patient at week 38, with a history of laparoscopic resection of the left fallopian tube performed 13 months before due to ectopic pregnancy was admitted to the Clinic on 5 August 2014 with initiating uterine contractions. According to the laparoscopy protocol the left fallopian tube was removed. The fallopian tube was excised from its uterine section with the use of electrocoagulation, without suturing. 4 months after the surgery the patient got pregnant again. The course of pregnancy was physiological. An obstetric examination performed in the admission room revealed regular uterine contractions with normal tension. No release of amniotic fluid was observed in clinical examination, although an ultrasound examination revealed a significant decrease of its amount. The general condition of the patient did not raise any doubts, and the pain did not go beyond symptoms of the first stage of labour. The patient was referred to a pre-delivery room to prepare her for a caesarean section due to a breach presentation in a nulliparous woman. In the peritoneal cavity, about $1,000 \mathrm{~mL}$ of clear fluid was found. The material was collected for a cytological analysis. A reproduction organ check-up revealed a non-bleeding disintegration of the uterine wall in the area of the left horn, at the scar after a removed fallopian tube, measuring about $1.5 \mathrm{~cm}$ in diameter, shown in Figure1. When a probe was inserted through the above-mentioned opening, it was freely moved into the uterine cavity (Fig. 2). The uterus was sewn up with single " $Z$ " sutures. The disrupted uterine wall was closed with three sutures of the same type, approximating the opening margins, which is also documented by photographs (Fig. 3). Early puerperium and post-operative period was uneventful. A cytological examination revealed cells corresponding to characteristic elements of amniotic fluid. A review of available literature does not give many publications that would present examples of uterine ruptures following laparoscopic procedures. It seems that laparoscopic salpingectomy is currently the best method of treatment of fallopian pregnancy but in this case, salpingectomy caused uterine rupture. Additionally, laparoscopic procedure was not accompanied by laying sutures, a thermal injury resulting from electrocoagulation weakened the uterine muscle. One element of the patient's admission to the Clinic that drew our attention was failure to notice free ascitic fluid during a transabdominal ultrasound examination. This could have resulted from inaccurate assessment of the upper abdominal organs during a standard ultrasound examination, or possibly from an uneven distribution of free fluid due to a horizontal position of the patient. In conclusion, it must be admitted that: pregnant women with a history of laparoscopic removal of ectopic pregnancy should be subject to special care. Resigning from suturing the uterine muscle may increase the risk of uterine rupture. Evaluation of the upper abdominal part should be an essential element of an ultrasound scans examination of patients with term pregnancies. Uterine rupture during pregnancy and labour may be asymptomatic.

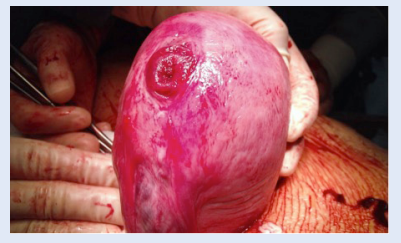

Figure 1. A non-bleeding rupture in the uterine wall, with the diameter of approx. $1.5 \mathrm{~cm}$

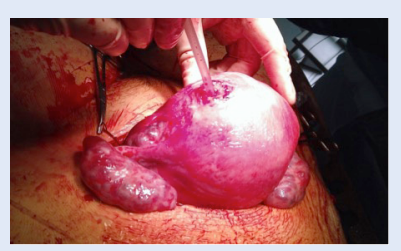

Figure 2. An instrument was passed through into the uterine cavity

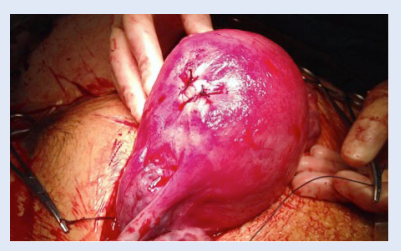

Figure 3. The uterus was sutured with single Z-type sutures

\section{Corresponding author:}

Anna Słomka

Department of Obstetrics, Gynecology and Gynecologic Oncology

Second Faculty of Medicine, Medical University of Warsaw

Kondratowicza 8, 03-242 Warszawa

e-mail: a.slomka@poczta.onet.eu 